\title{
Análisis biomecánico de fracturas subtrocantéreas femorales. Factores críticos para una adecuada consolidación.
}

\author{
Jorge Rosell Pradas ${ }^{1}$, José Manuel Naveiro Gomez ${ }^{1}$, Carmen Martínez Aznar ${ }^{2}$ \\ ${ }^{1}$ Afiliación: Grupo Biomateriales \\ Instituto de Investigación en Ingeniería de Aragón (I3A) \\ Uiversidad de Zaragoza, Mariano Esquillor s/n, 50018, Zaragoza, Spain. \\ Tel. +34-976762707, e-mail: 680476@unizar.es \\ ${ }^{2}$ Afiliación: Hospital Universitario Miguel Servet, Zaragoza
}

\section{Resumen}

Las fracturas subtrocantéreas no son las más frecuentes, pero presentan una mayor complejidad en su tratamiento quirúrgico, sin que existan criterios claros para escoger un tratamiento óptimo [1]. Este estudio plantea un análisis biomecánico de este tipo de fracturas tratadas mediante clavos intramedulares con tornillo cefálico.

\section{Materiales y métodos}

Este estudio plantea el análisis biomecánico una fractura subtrocantérea transversa en zona 2 de Wiss, con gaps de fractura (separación entre superficies de fractura) de 3,6 y $10 \mathrm{~mm}$ respectivamente, analizando el efecto que tiene la elección de material del clavo y los tornillos, acero inoxidable o titanio, y el tamaño de gap de fractura en los parámetros más críticos para una adecuada consolidación, como son la estabilidad global de la osteosíntesis y los movimientos en el foco de fractura. Para ello se han creado 3 modelos distintos de elementos finitos.

El modelo tridimensional del fémur ha sido creado utilizando el Escáner Láser 3D Roland Picza, con una resolución de 0,2 mm, obteniendo un modelo geométrico realmente preciso a partir de una réplica anatómica de un fémur sano. Los modelos tridimensionales del clavo Gamma y los tornillos se han creado de forma manual con el software de diseño 3D NX-Ideas, el mismo software que se usa para realizar el proceso de mallado [2]. Los tornillos cefálicos y distales se han modelado como cilindros simples.

La malla se realiza mediante elementos tipo tetraedro con aproximación lineal y un tamaño adecuado para obtener la precisión deseada, caracterizado mediante un análisis de sensibilidad. Los modelos desarrollados se muestran en la Figura 1.
Se considera un comportamiento elástico, lineal e isótropo para todos los materiales (hueso cortical, hueso trabecular, y acero inoxidable o titanio para el clavo intramedular y los tornillos cefálico y distal).

Las condiciones de contorno son las mismas para los 3 modelos. En cuanto a desplazamientos, se bloquea el movimiento del área condilar femoral. En cuanto a las cargas, se considera un apoyo accidental del pie en el suelo en la etapa postoperatoria temprana, con una carga equivalente al $25 \%$ de la carga fisiológica máxima, tanto en la cabeza femoral como en el trocánter mayor, como puede verse en la figura 2.

Finalmente se definen las interacciones entre las partes del modelo, estableciendo las condiciones de contacto entre el clavo y los tornillos cefálico y distal, $\mathrm{y}$ entre ellos y el hueso.

El proceso de cálculo y la visualización de los resultados se realiza con el software ABAQUS [3].

Todo el proceso de desarrollo e implementación de los modelos se detalla en [4].

\section{Resultados}

Los clavos de titanio presentan un desplazamiento máximo en la cabeza femoral un 33\% mayor que los clavos de acero inoxidable, Los clavos de titanio acrecientan el desplazamiento medio del foco de fractura un 57\% de media en comparación con el acero inoxidable. Con respecto al tamaño del gap de fractura, su influencia apenas es apreciable cuando se comparan valores de desplazamientos máximo en la cabeza femoral, mientras que se aprecia un incremento de $2 \%$ en el desplazamiento medio del foco de fractura al comparar los modelos con la fractura de $3 \mathrm{~mm}$ frente a la de $6 \mathrm{~mm}$, y un incremento de $4.5 \%$ en el desplazamiento medio al comparar los modelos con fractura de $3 \mathrm{~mm}$ frente a la de $10 \mathrm{~mm}$. Ver figuras 3 y 4 . 


\section{Conclusiones}

Ambos materiales proporcionan suficiente estabilidad en las fracturas analizadas, si bien el titanio da lugar a osteosíntesis menos rígidas que permiten un mayor movimiento en el foco de fractura, lo que dentro de un cierto umbral estimulará la regeneración ósea. Mecánicamente se ha comprobado que el incremento del gap de fractura no influye en gran medida a la estabilidad global o a la movilidad del foco de fractura, mientras que clínicamente es algo de gran relevancia. Clínicamente el un gap de fractura superior complica la reducción quirúrgica de la fractura, llegando al extremo de necesitar injertos óseos en fracturas muy conminutas; este parámetro ha demostrado ser uno de los más críticos con relación al tiempo de consolidación de la fractura. La elección de material del clavo y los tornillos en la práctica clínica suele depender de cada cirujano, de su experiencia con cada tipo de implante y del caso en concreto.

\section{Figuras}

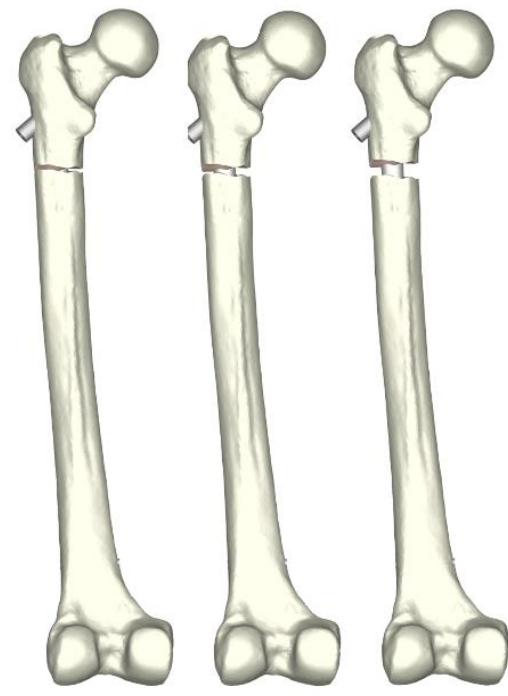

Figura 1. Modelos de osteosíntesis con gaps de 3, 6 y $10 \mathrm{~mm}$.

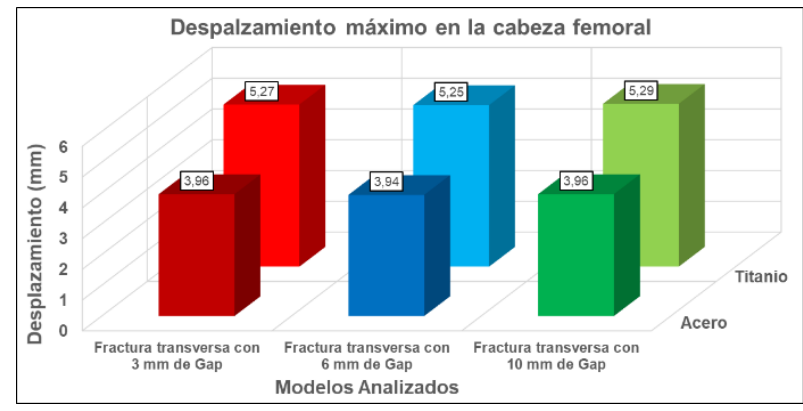

Figura 3. Desplazamiento máximo en la cabeza femoral. Resultados obtenidos en los análisis para los tres modelos creados, analizados con acero y titanio como material para el clavo y los tornillos.

\section{Referencias}

[1]. Ng Ac, Drake Mt, Clarke Bl, Sems Sa, Atkinson Ej, Achenbach $\mathrm{Sj}$, Melton $\mathrm{Lj}$ 3rd. Trends in subtrochanteric, diaphyseal, and distal femur fractures, 1984-2007. Osteoporos Int. 2012 Jun;23(6):1721-6. doi: 10.1007/s00198-011-1777-9. Epub 2011 Sep 10. PMID: 21909727; PMCID: PMC3266989.FRÖHLICH, B. and PLATE, J. The cubic mouse: a new device for three-dimensional input. In TURNER, T. and SZWILLUS, G. (eds.). Proceedings of the SIGCHI Conference on Human Factors in Computing Systems. New York: ACM, 2000, pp. 526-531.

[2]. Siemens, I-deas ${ }^{\circledR} 11$ NX Series PLM software, 2020. [https://www.plm.automation.siemens.com].

[3]. Dassault Systèmes, 2020. [https://www.3ds.com].

[4]. Herrera A, Rosell J, Ibarz E, Albareda J, Gabarre S, Mateo J, Gracia L. Biomechanical analysis of the stability of anterograde reamed intramedullary nails in femoral spiral fractures. Injury. 2020 Apr;51 Suppl 1:S74-S79. doi: 10.1016/j.injury.2020.02.034. Epub 2020 Feb 18. PMID: 32081396.

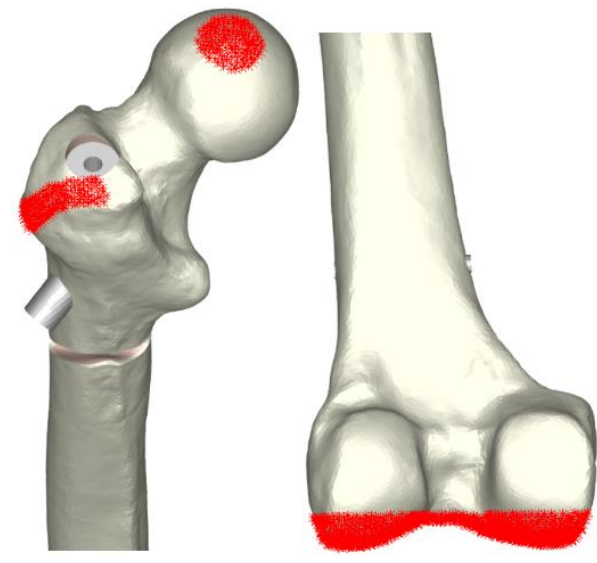

Figura 2. Condiciones de contorno. Áreas de aplicación de las fuerzas en la cabeza femoral y en el trocánter mayor, lado izquierdo. Área condilar bloqueada, lado derecho.

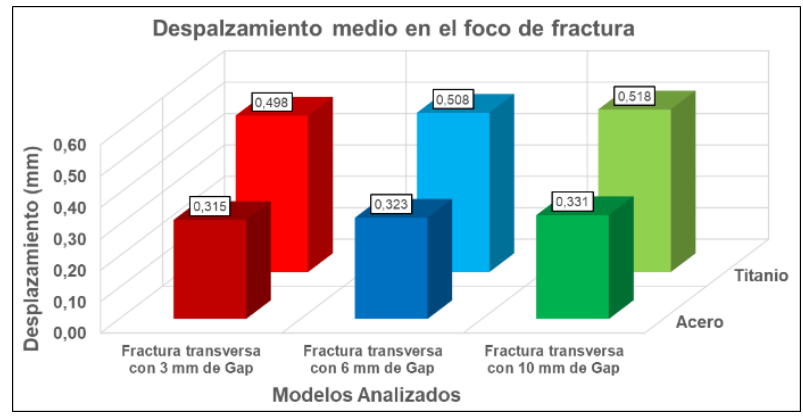

Figura 4. Desplazamiento medio en el foco de fractura. Resultados obtenidos en los análisis para los tres modelos creados, analizados con acero y titanio como material para el clavo y los tornillos.

Revista “Jornada de Jóvenes Investigadores del I3A”, vol. 8 (Actas de la IX Jornada de Jóvenes Investigadores del I3A -11 de diciembre de 2020). ISSN 2341-4790. 